\title{
Intermodal interferometer for strain and temperature sensing fabricated in birefringent boron doped microstructured fiber
}

\author{
G. Statkiewicz-Barabach, ${ }^{1, \star}$ J. P. Carvalho, ${ }^{2}$ O. Frazão, ${ }^{2}$ J. Olszewski, ${ }^{1}$ \\ P. Mergo, ${ }^{3}$ J. L. Santos, ${ }^{2}$ and W. Urbanczyk ${ }^{1}$ \\ 'Wroclaw University of Technology, Institute of Physics, Wybrzeze Wyspianskiego 27, 50-370 Wroclaw, Poland \\ ${ }^{2}$ INESC Porto, Rua do Campo Alegre 687, 4169-007, Porto, Portugal \\ ${ }^{3}$ Maria Curie-Sklodowska University, PI. Marii Curie-Sklodowskiej 5, 20-031 Lublin, Poland \\ *Corresponding author: Gabriela.Statkiewicz@pwr.wroc.pl
}

Received 1 February 2011; revised 17 May 2011; accepted 23 May 2011;

posted 25 May 2011 (Doc. ID 141936); published 11 July 2011

\begin{abstract}
We present a compact in-line fiber interferometric sensor fabricated in a boron doped two-mode highly birefringent microstructured fiber using a $\mathrm{CO}_{2}$ laser. The intermodal interference arises at the fiber output due to coupling between the fundamental and the first order modes occurring at two fiber tapers distant by a few millimeters. The visibility of intermodal interference fringes is modulated by a polarimetric differential signal and varies in response to measurand changes. The proposed interferometer was tested for measurements of the strain and temperature, respectively, in the range of $20-700{ }^{\circ} \mathrm{C}$ and $0-17$ mstrain. The sensitivity coefficients corresponding to fringe displacement and contrast variations are equal respectively for strain $-2.51 \mathrm{~nm} / \mathrm{mstrain}$ and $-0.02561 / \mathrm{mstrain}$ and for temperature $16.7 \mathrm{pm} /{ }^{\circ} \mathrm{C}$ and $5.74 \times 10^{-5} 1 /{ }^{\circ} \mathrm{C}$. This allows for simultaneous measurements of the two parameters by interrogation of the visibility and the displacement of interference fringes. (C) 2011 Optical Society of America
\end{abstract}

OCIS codes: $\quad 060.2370,060.4005,120.3180$.

\section{Introduction}

Microstructured optical fibers (MOFs) have been widely studied in recent years owing to their potential for obtaining novel transmission and nonlinear and sensing characteristics. A possible range of sensing applications of MOFs covers various types of devices for measurements of different physical parameters [1-4] and specific chemical compounds in gases and liquids, employing evanescent field techniques [4-6] and direct overlap of the guided mode with the analyte filling the hollow core in photonic bandgap fibers [7]. It has also been demonstrated that MOFs can be used as active and passive

0003-6935/11/213742-08 $\$ 15.00 / 0$

(C) 2011 Optical Society of America elements in fiber-optic polarimetric and interferometric sensors [요, 9$]$.

Optical fiber sensors based on intermodal interference between core and cladding modes have been proposed in recent years and tested for various applications [10-15]. Such interferometric sensors have several advantages over other sensing concepts, including small size, high sensitivity and resolution, fast response time, and low cost. Different solutions to obtain all-fiber intermodal interference in microstructured fibers are reported in published literature. One of them is to splice a short piece of the microstructured fiber between two pieces of the same fiber [10] or standard single mode fiber [11-13]. The other possibility is to make a tapered zone [14], collapse the holes of the MOF at two different places $[13,15]$ or to splice two pieces of MOFs with a small offset [15]. In the last approach, different higher 
order modes can be excited by tuning the lateral offset of the spliced fibers.

In this Paper, we demonstrate an in-line fiber intermodal interferometer fabricated in a boron doped highly birefringent microstructured fiber. The boron doped region located in the middle of the core decreases the effective index of the fundamental mode and facilitates coupling between the fundamental and the first order mode. The coupling regions have the form of tapers fabricated using $\mathrm{CO}_{2}$ lasers and are distant by a few millimeters. Light from a broadband source is coupled into the fundamental $\mathrm{LP}_{01}$ mode of the microstructured fiber. At the fiber output, the light confined mostly in the fundamental mode is delivered to the optical spectrum analyzer by leading-out fiber. As a result, the spectral intensity at the sensor output is modulated only by intermodal interference produced by a short piece of fiber between the two coupling points. Moreover, as the fiber is highly birefringent, each pair of polarization modes produces its own intermodal fringes, which results in the contrast modulation of the overall interference signal observed at the fiber output. On the other hand, it provides an additional degree of freedom in interrogating the interference signal and makes it possible to measure simultaneously two physical parameters acting on the interferometer.

The typical limitations of the MOFs based interferometric sensors are related to the efficiency in optical power launching, difficulties in controlling light polarization, the need for unavailable MOFs based passive devices, and/or incompatibility with standard fibers. The proposed sensor deals with almost all of these impairments, except the difficulty in polarization control, which was overcome thanks to high fiber birefringence giving rise to contrast modulation of the intermodal interference fringes when the sensor is powered with depolarized light. To the best of our knowledge, this is the first intermodal highly birefringent MOFs based interferometric sensor reported in literature. In comparison with the fiber Bragg grating sensors, it shows two times greater sensitivity to strain and temperature. Moreover, the proposed sensor allows for simultaneous measurements of the two parameters by interrogation of the visibility and the displacement of interference fringes.

\section{Sensor Fabrication and Operation Principle}

For fabrication of the intermodal interferometer, we have used a boron doped birefringent microstructured fiber shown in Fig. 1, which was drawn at the Department of Optical Fibers Technology, University of Marie Curie-Sklodowska in Lublin, Poland. The birefringence in this fiber is induced by two large holes located symmetrically with respect to the core. Its geometrical parameters averaged for the first two layers of holes surrounding the core are as follows: pitch distance $3.6 \mu \mathrm{m}$, diameter of cladding holes $1.63 \mu \mathrm{m}$, diameter of large holes $4.14 \mu \mathrm{m}$, external diameter of the fiber $100 \mu \mathrm{m}$, size of the boron doped inclusion $0.46 \times 0.79 \mu \mathrm{m}$, and boron concentration in the inclusion $13 \mathrm{~mol} \%$. It is worth mentioning that fibers of similar construction with properly optimized geometry can be used as a wideband fiberoptic polarizer [16].

To better understand the behavior of the proposed sensor, we modeled propagation characteristics of the boron doped fiber using a fully vectorial mode solver based on the hybrid edge-nodal finite-element method (FEM) with a perfectly matched layer [17]. As it can be seen in Fig. 1, the cross section of the investigated fiber is not uniform due to technological imperfections. The edges of the holes in the cladding and the doped inclusion were automatically detected by post processing of the scanning electron microscope (SEM) image, which included modification of the histogram followed by thresholding and binarization. Using this tool, we could generate the mesh for FEM calculations, which reflected the actual shape and location of each hole with an accuracy of about $30 \mathrm{~nm}$. A precise copying of the fiber geometry is a key requirement for accurate modeling of its propagation characteristics. Using such an approach, we calculated the spectral dependence of effective indices for the fundamental and the first order polarization modes, phase and group modal birefringence and the confinement losses for each mode. Numerical

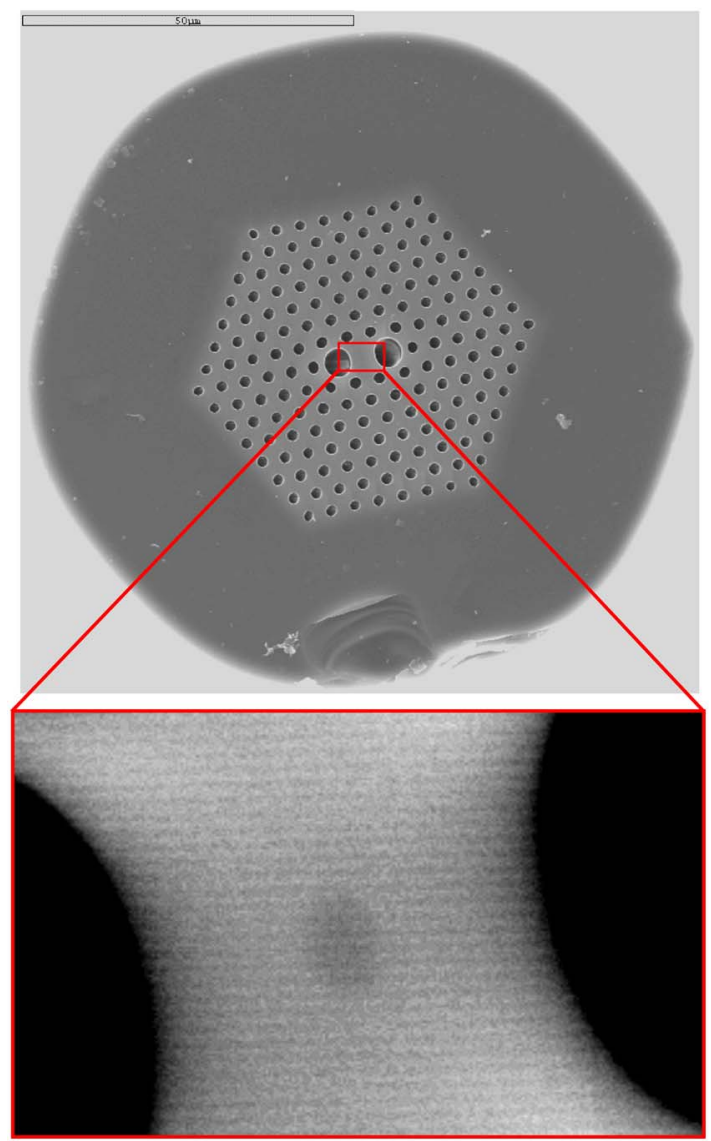

Fig. 1. (Color online) SEM image of the birefringent MOF with boron doped inclusion in the center of the core (darker spot) used for fabrication of the intermodal interferometer. 
calculations presented in Fig. 2 clearly prove that the boron doped inclusion diminishes the effective index of the fundamental mode, while the first order mode remains unaffected. This can be intuitively understood on the grounds of the perturbation theory [18], which predicts that the change in the effective index squared for a specific mode can be expressed as the following overlap integral:

$$
\delta n_{\mathrm{eff}}^{2}=\int \delta n^{2}|\Psi|^{2} \mathrm{~d} A
$$

where $|\Psi|^{2}$ represents the normalized intensity distribution in the considered mode and $\delta n^{2}$ stands for the change in the material refractive index squared introduced by the inclusion. As it is shown in Fig. 2, the overlap integral between the first order mode and the inclusion is close to zero, while for the fundamental mode it takes the highest value. This explains the difference in the impact of the inclusion on the fundamental and the first order mode. Moreover, lower effective index of the fundamental mode causes it to spread deeper into the microstructured cladding in the tapered regions, which facilitates its coupling to the $\mathrm{LP}_{11}$ mode.

The operation principle of the proposed interferometer is schematically illustrated in Fig. 3(a), while in Fig. 3(b) we show two tapered regions formed by exposing the fiber to a $\mathrm{CO}_{2}$ laser beam and simultaneously stretching it when the glass becomes soft. The first taper causes the coupling of the $\mathrm{LP}_{01}$ to the $\mathrm{LP}_{11}$ mode, and the two modes copropagate beginning from this point. At the second taper the two modes are recombined producing the interference signal. The $\mathrm{LP}_{11}$ mode is partially filtered out at the splice between the boron doped two-mode microstructured fiber and the endlessly single modes leading-out fiber (not shown in Fig. 3). As a result, at the sensor output we observed only the interference signal carried in the fundamental mode. Such an inline fiber Mach-Zehnder interferometer is very compact and easy to fabricate. The splices have been made using a fusion arc splicer. Special care was taken to minimize the collapse of the microstructured regions. A typical loss of about $3 \mathrm{~dB}$ was achieved at each splice.

For a depolarized broad band input beam, at the fiber output we observe superposition of two interference signals produced by two pairs of the orthogonally polarized modes. Assuming that the coupling strength is the same for both polarizations, one obtains the following expressions for the interference signal produced by the modes $\mathrm{LP}_{01}{ }^{x}$ and $\mathrm{LP}_{11}{ }^{x}$

$$
I_{x}(\lambda)=I_{0}\left(1+\gamma \cos \frac{2 \pi\left(n_{01}^{x}-n_{11}^{x}\right) L}{\lambda}\right)
$$

and respectively by the modes $\mathrm{LP}_{01}{ }^{y}$ and $\mathrm{LP}_{11}{ }^{y}$

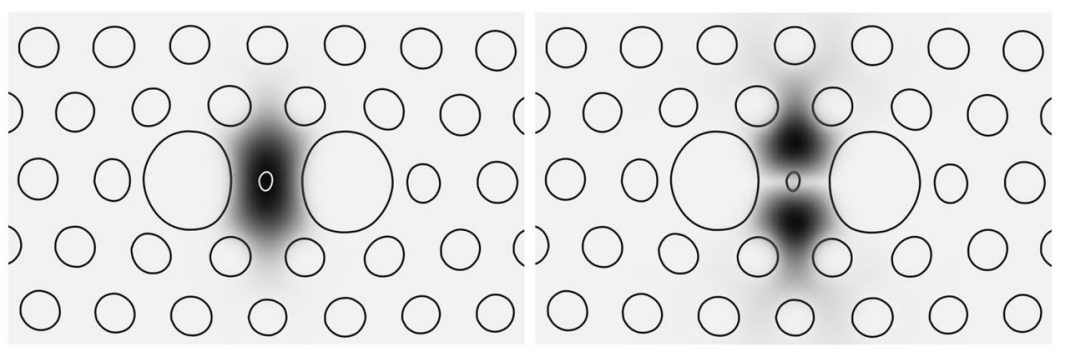

(a)

(b)

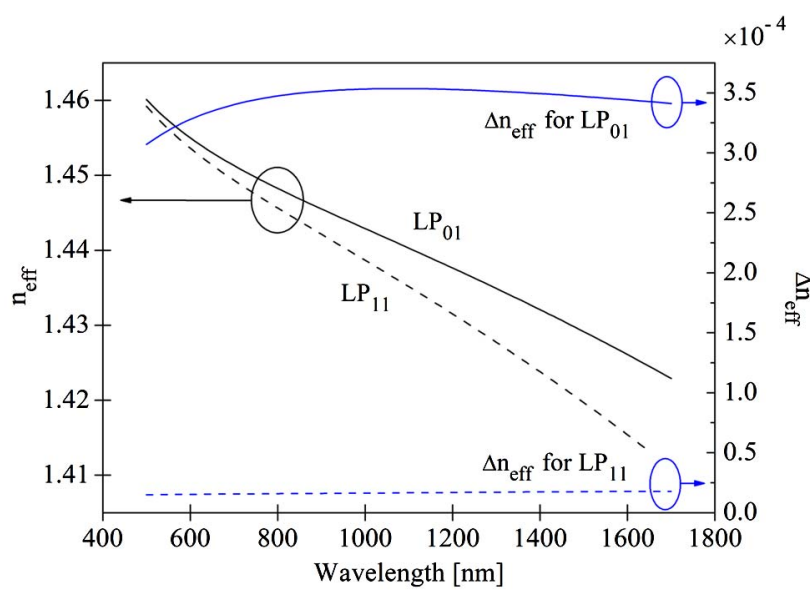

(c)

Fig. 2. (Color online) Field distribution calculated for the fundamental (a) and the first order mode (b) at $\lambda=1.55 \mu \mathrm{m}$. Spectral dependence of the effective indices in the fiber with boron doped inclusion for the fundamental and the first order modes and the effective index change introduced by the inclusion (c). 
(a)

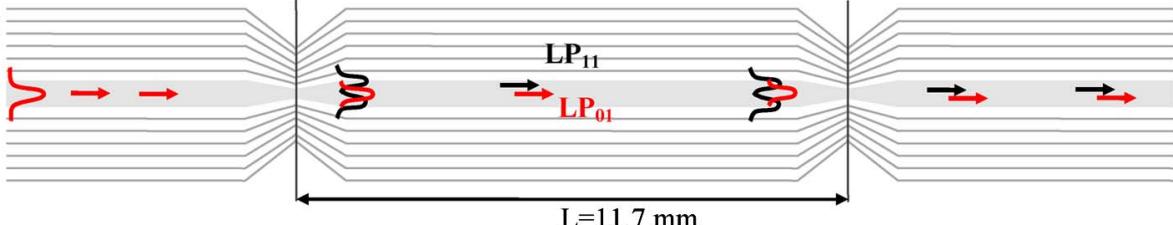

(b)

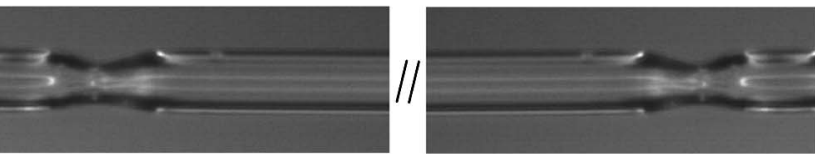

Fig. 3. (Color online) Schematic configuration of the proposed intermodal interferometric sensor (a) and the microscope image of the microstructured fiber with the interferometer formed by two tapered regions (b).

$$
I_{y}(\lambda)=I_{0}\left(1+\gamma \cos \frac{2 \pi\left(n_{01}^{y}-n_{11}^{y}\right) L}{\lambda}\right)
$$

where $\gamma$ is the fringe contrast. In the output spectrogram, we observe the superposition of the two interference signals, which can be represented by the following formula:

$$
\begin{aligned}
I(\lambda)= & 2 I_{0}\left[1+\gamma \cos \left(\frac{\pi\left(n_{01}^{x}+n_{01}^{y}-n_{11}^{x}-n_{11}^{y}\right) L}{\lambda}\right)\right. \\
& \left.\cos \left(\frac{\pi\left(n_{01}^{x}-n_{01}^{y}-n_{11}^{x}+n_{11}^{y}\right) L}{\lambda}\right)\right] .
\end{aligned}
$$

The above equation can be represented as:

$$
\begin{aligned}
I(\lambda)= & 2 I_{0}\left[1+\gamma \cos \left(\frac{2 \pi\left(n_{01}^{a}-n_{11}^{a}\right) L}{\lambda}\right)\right. \\
& \left.\cos \left(\frac{\pi\left(\Delta n_{01}-\Delta n_{11}\right) L}{\lambda}\right)\right]
\end{aligned}
$$

where $n_{01}{ }^{a}$ and $n_{11}{ }^{a}$ stands respectively for the effective refractive index of the fundamental and the first order mode averaged with respect to polarization:

$$
n_{01}^{a}=\frac{n_{01}^{x}+n_{01}^{y}}{2}, \quad n_{11}^{a}=\frac{n_{11}^{x}+n_{11}^{y}}{2}
$$

and $\Delta n_{01}$ and $\Delta n_{11}$ stands for the phase modal birefringence in the fundamental and the first order mode

$$
\Delta n_{01}=n_{01}^{x}-n_{01}^{y}, \quad \Delta n_{11}=n_{11}^{x}-n_{11}^{y} .
$$

The first cosine term in Eq. (5) represents the intermodal interference fringes averaged with respect to polarization, while the second term constitutes the slowly varying envelope determined by the birefringence difference in the fundamental and the first order mode. The corresponding terms are indicated by black and red lines in Fig. 4, which shows the output interferogram produced by the $11.7 \mathrm{~mm}$ long interferometer.

To confirm that the coupling indeed occurs between the fundamental and the first order mode, we have compared the calculated and measured difference in the group refractive indices for these modes averaged with respect to polarization $N_{01}^{a}-N_{11}^{a}$. It is well known [19] that the difference $N_{01}^{a}-N_{11}^{a}$ can be determined from spectral interference fringes shown in Fig. $\underline{4}$ :

$$
N_{01}^{a}-N_{11}^{a}=\frac{\lambda^{2}}{\Delta \lambda L},
$$

where $L=11.7 \mathrm{~mm}$ is the interferometer length and $\Delta \lambda$ is the separation of successive interference fringes. The measured and calculated values of $N_{01}^{a}-$ $N_{11}^{a}$ presented in Fig. 5 show very good agreement. We compared also the difference in group modal birefringence between the fundamental and the first order mode $\left(\Delta N_{01}-\Delta N_{11}\right)$ calculated and estimated from the spectrogram shown in Fig. 4. For experimental evaluation of the birefringence difference, we used the following relation:

$$
\Delta N_{01}-\Delta N_{11}=\frac{\lambda^{2}}{2 \Delta \lambda L},
$$

where $\Delta \lambda$ indicates the separation of successive contrast extremes (maxima or minima). The numerical values of the group birefringence were obtained from FEM analysis. The positions of minima and maxima of the interference pattern envelope were approximated by a red curve shown in Fig. $\underline{4}$ with precision

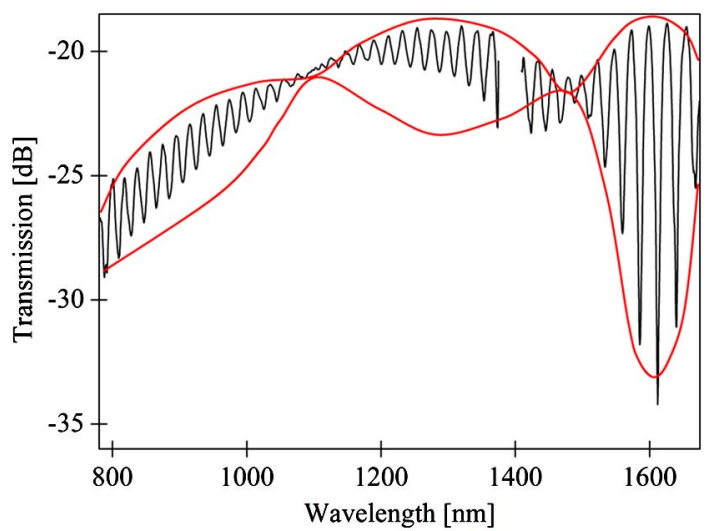

Fig. 4. (Color online) The interference spectrum at the output of an in-fiber Mach-Zehnder intermodal interferometer fabricated in the microstructured fiber. 


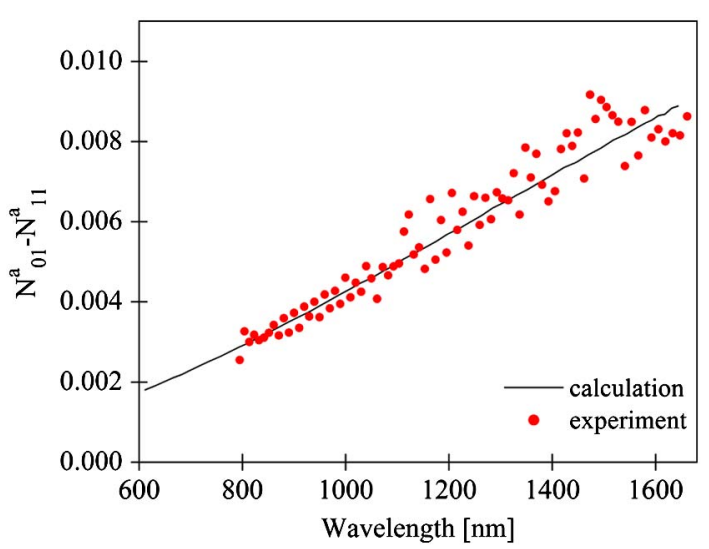

Fig. 5. (Color online) Calculated (solid line) and measured (dots) difference in group effective indices of the $\mathrm{LP}_{01}$ and $\mathrm{LP}_{11}$ modes averaged with respect to polarization.

of about $15 \mathrm{~nm}$. In Fig. 6 we present the calculated values of the birefringence difference $\left(\Delta N_{01}-\Delta N_{11}\right)$ and compare them with the experimental values estimated from the spectrogram with precision of about $25 \%$. Good agreement between the simulation

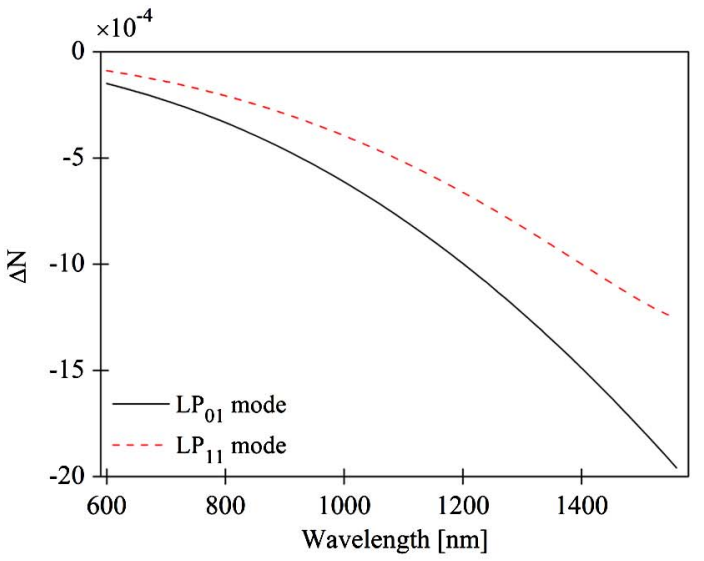

(a)

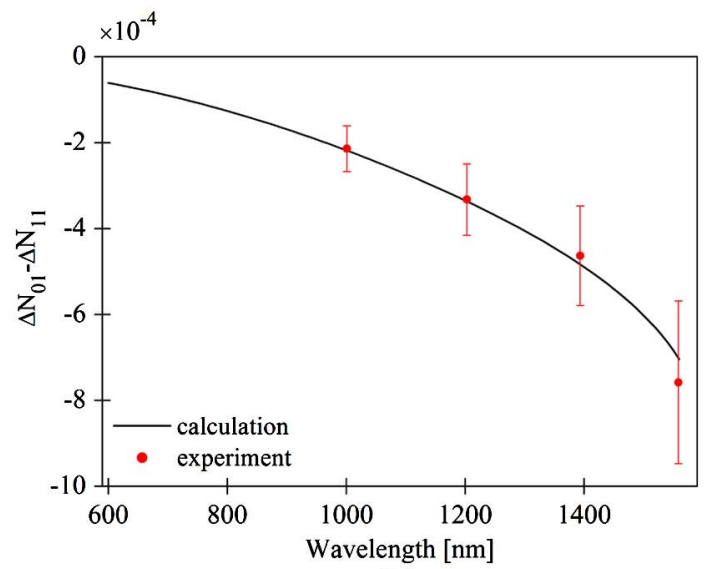

(b)

Fig. 6. (Color online) Calculated group modal birefringence of the $\mathrm{LP}_{01}$ (solid line) and $\mathrm{LP}_{11}$ (dashed line) modes in the investigated fiber with boron doped inclusion (a) and comparison of the calculated (solid line) and measured (dots) birefringence difference (b). results and measured values of $\left(\Delta N_{01}-\Delta N_{11}\right)$ provides additional argument that the observed fringes result from the interference between the $\mathrm{LP}_{01}$ and $\mathrm{LP}_{11}$ modes.

\section{Experimental Results and Discussion}

The experimental setup for measurement of different physical parameters using the proposed interferometer is presented in Fig. 7. As a light source we used an amplified spontaneous emission source with a central wavelength at $1570 \mathrm{~nm}$. The transmission spectrum at the output of the interferometer was registered by an optical spectrum analyzer with resolution of $0.05 \mathrm{~nm}$.

As it is shown in Fig. 8, the interference fringes with the modulation depth increasing against the wavelength were observed in the spectral range of $1520-1620 \mathrm{~nm}$. Variation of both the modulation depth and the fringe displacement were observed in response to changes in temperature and strain applied to the interferometer.

According to Eq. (5), the shift of interference fringes is related to the variation of the effective refractive indices of the fundamental and the first order mode $n_{01}{ }^{a}$ and $n_{11}{ }^{a}$ and the interferometer length $L$. The shift of the interference fringe $\Delta \lambda$ induced by the measurand change $\Delta X$ can be quantitatively represented by the following relation:

$$
\frac{\partial}{\partial \lambda}\left(\frac{\left(n_{01}^{a}-n_{11}^{a}\right) L}{\lambda}\right) \Delta \lambda+\frac{\partial}{\partial X}\left(\frac{\left(n_{01}^{a}-n_{11}^{a}\right) L}{\lambda}\right) \Delta X=0 .
$$

After performing rather straightforward calculations, one can express the sensitivity of the proposed sensors in the following way:

$$
\frac{1}{\lambda} \frac{\Delta \lambda}{\Delta X}=\frac{n_{01}^{a}-n_{11}^{a}}{N_{01}^{a}-N_{11}^{a}}\left(\frac{1}{L} \frac{\partial L}{\partial X}+\frac{1}{n_{01}^{a}-n_{11}^{a}} \frac{\partial\left(n_{01}^{a}-n_{11}^{a}\right)}{\partial X}\right)
$$

or more conveniently with relation to the phase intermodal sensitivity of the fiber:

$$
\frac{\Delta \lambda}{\Delta X}=\frac{\lambda^{2} K_{X}}{2 \pi\left(N_{01}^{a}-N_{11}^{a}\right)},
$$

where

$$
K_{X}=\frac{1}{L} \frac{d\left(\varphi_{01}^{a}-\varphi_{11}^{a}\right)}{d X}
$$

temperature, strain

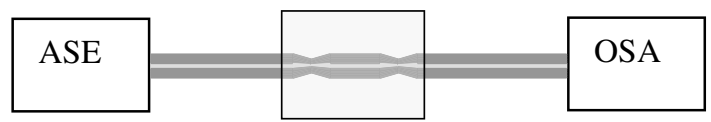

Fig. 7. Experimental setup for strain and temperature measurements. 


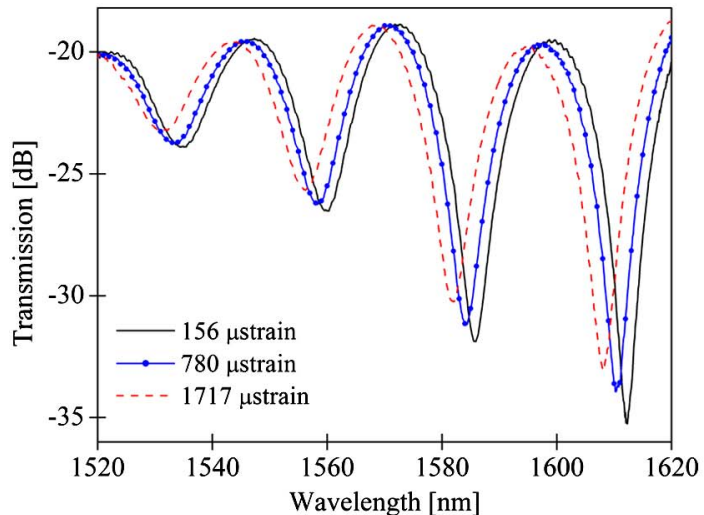

(a)

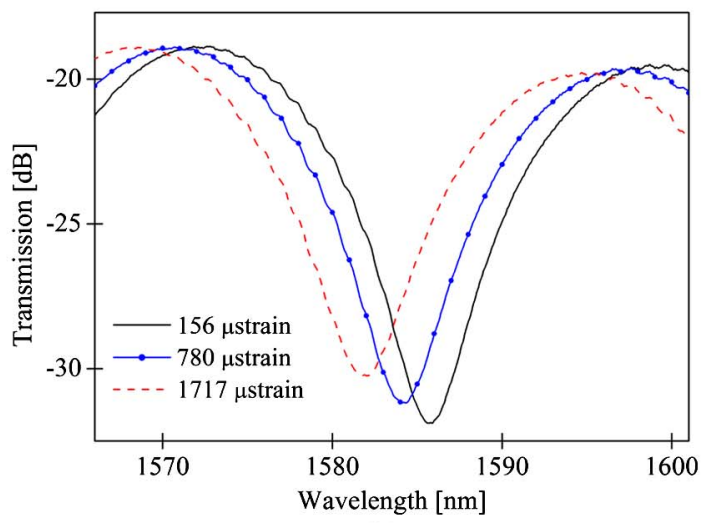

(b)

Fig. 8. (Color online) Transmission characteristic registered in the full spectral range for selected values of applied strain (a) and variation of the modulation depth and displacement of the third fringe in response to applied strain (b).

and $\varphi_{01}^{a}$ and $\varphi_{11}^{a}$ are the phase shifts averaged with respect to polarization induced by the unit change in the measurand in the fiber of the unit length. On the other hand, to avoid ambiguity, the measurandinduced displacement of the interference fringe must be lower than the separation of successive fringes defined by Eq. (8). This leads to a simple relation between the maximum length of the sensor and its operation range

$$
L_{\max } \Delta X_{\max }<\frac{2 \pi}{K_{X}} .
$$

For the sake of simplicity we considered that the interferometer length is comprised between the narrowest points in the tapered regions. As the taper's length (about $150 \mu \mathrm{m}$ ) is relatively small compared to the interferometer length $(10-20 \mathrm{~mm})$, we disregarded the effect of the transition regions on variations of $K_{X}$ and assumed that the fiber sensitivity is constant over the entire sensor length.

In strain measurements, the interferometer was elongated up to 1.7 mstrain, while for temperature tests it was placed in an electrical oven, whose temperature was increased from $20^{\circ} \mathrm{C}$ to $700^{\circ} \mathrm{C}$. The precision of temperature control in the oven was about
$5{ }^{\circ} \mathrm{C}$. In spite of a wide temperature range, we did not observe any changes in the sensor characteristics after several temperature cycles. A possible impact of the boron diffusion on the sensor performance is minimized by the fact that the boron doped inclusion is located in the center of the core. Therefore, a change in the boron concentration profile has little influence on the overlap coefficient with the fundamental and the first order modes, which according to the perturbation approach represent the impact of the diffusion process on the effective indices of both modes. It is however possible that in more demanding applications the boron diffusion may limit the maximum temperature range of the proposed sensor.

As it is shown in Fig. 8, the interference fringes move toward shorter wavelengths and their visibility decreases in response to the applied strain. The displacement of the third fringe with the intensity minimum located at $1583 \mathrm{~nm}$, is linear in the investigated range of strain $0-1.7$ mstrain with no trace of hysteresis. The sensitivity coefficient for strain is $-2.51 \mathrm{~nm} / \mathrm{mstrain}$, see Fig. 9(a). Moreover, the visibility of the third fringe [Fig. 9(b)] linearly decreases against the applied strain with a rate of $-0.02561 /$ mstrain. The root mean square deviation of the experimental data from a linear trend is equal

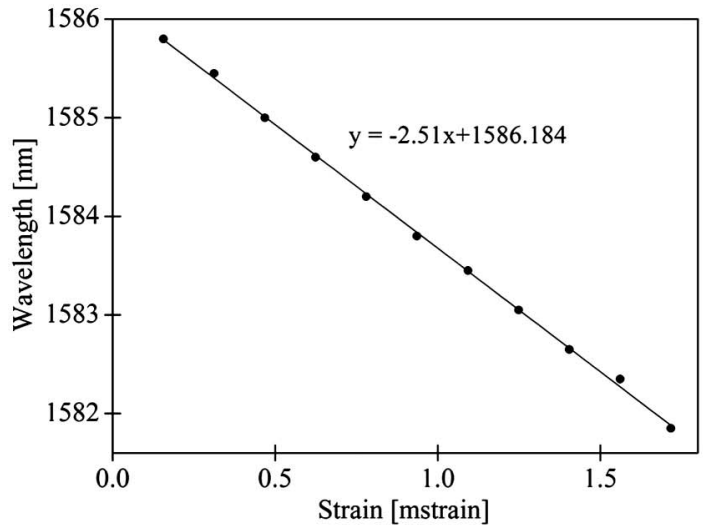

(a)

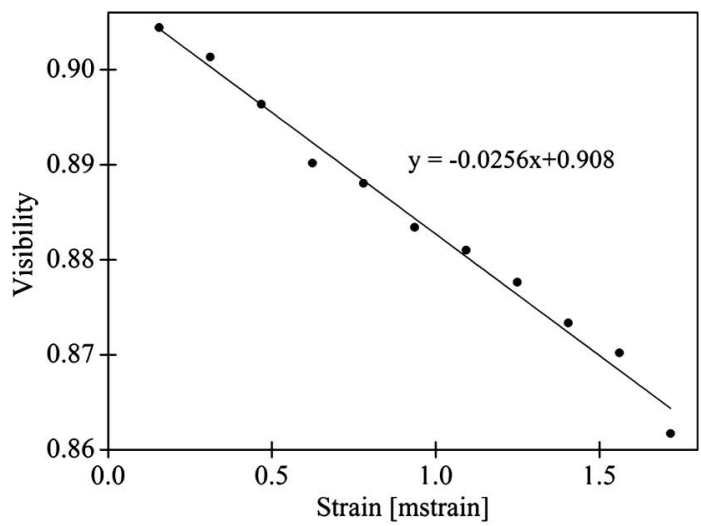

(b)

Fig. 9. Displacement of the third interference fringe against applied strain (a) and change in its visibility (b). 
respectively to $35 \mathrm{pm}$ for the fringe displacement and $1.4 \times 10^{-3}$ for the visibility change.

In Fig. 10(a), we present the measured linear dependence of the third fringe displacement induced by temperature change. The resulting sensitivity to temperature has a positive sign and equals $16.7 \mathrm{pm} /{ }^{\circ} \mathrm{C}$. The visibility of the third fringe linearly increases against temperature, which results in a sensitivity coefficient equal to $5.74 \times 10^{-5} 1 /{ }^{\circ} \mathrm{C}$. In this case the root mean square deviation of the experimental data from a linear trend is $76 \mathrm{pm}$ for the fringe displacement and $1.7 \times 10^{-3}$ for visibility variation. As the sensitivity coefficients for strain and temperature have opposite signs and are different in absolute values, the sensitivity matrix for these two parameters is well conditioned, thus providing a possibility of simultaneous measurements of strain and temperature by interrogating the position and the visibility of a selected fringe.

In terms of interferometric sensitivity, the performance of the proposed sensor is similar to other intermodal MOFs based sensors reported in literature $[15,20,21]$ For example, the sensitivity to strain reported for nonbirefringent MOFs ranges from $2.28 \mathrm{pm} / \mu$ strain [15] to $5.3 \mathrm{pm} / \mu$ strain [20], while the sensitivity to temperature is $12 \mathrm{pm} /{ }^{\circ} \overline{\mathrm{C}}[20]$ and $3-5 \mathrm{pm} /{ }^{\circ} \mathrm{C}$ [21]. These numbers do not differ significantly from the interferometric sensitivity of the pro-

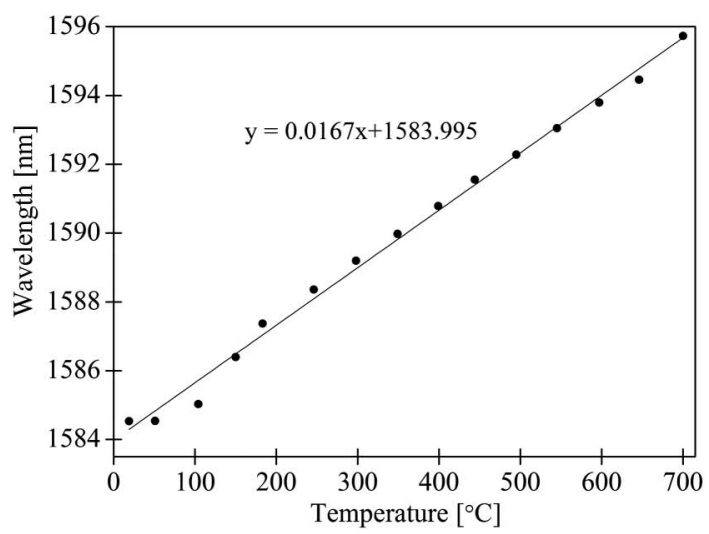

(a)

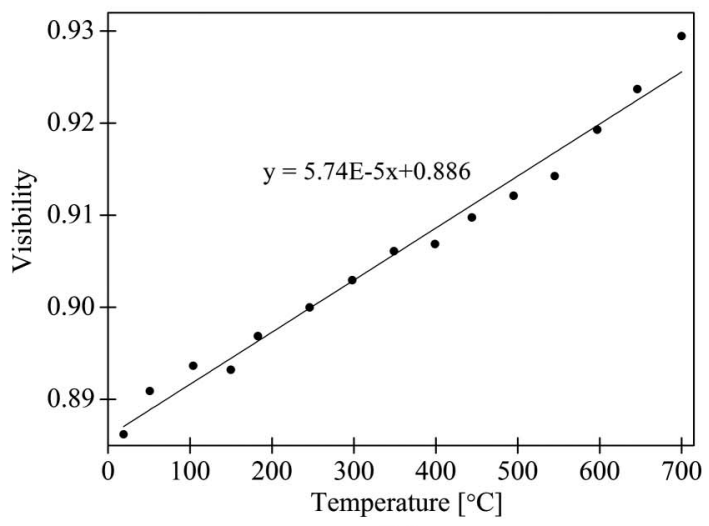

(b)

Fig. 10. Displacement of the third interference fringe against temperature (a) and change of its visibility (b). posed sensors, which is equal to $-2.51 \mathrm{pm} / \mu$ strain and $16.7 \mathrm{pm} /{ }^{\circ} \mathrm{C}$, respectively. However, the main advantage of the proposed sensor is that thanks to high birefringence of the MOF one can interrogate simultaneously the displacement of the interference fringes and the contrast variations.

\section{Conclusions}

In this work, we demonstrate the in-line fiber MachZehnder interferometer for strain and temperature measurements, fabricated in a highly birefringent boron doped microstructured fiber using a $\mathrm{CO}_{2}$ laser. The operation principle of the proposed sensor exploits the effect of intermodal interference arising between the fundamental $\mathrm{LP}_{01}$ and the first order $\mathrm{LP}_{11}$ mode. Boron doped inclusion facilitates coupling between the two modes, which takes place in the fiber tapers fabricated using $\mathrm{CO}_{2}$ laser. The origin of the interference fringes and contrast modulation has been confirmed by comparing the measured and calculated values of group effective indices and group birefringence for the $\mathrm{LP}_{01}$ and the $\mathrm{LP}_{11}$ modes. $\mathrm{Be}-$ cause of high fiber birefringence, the intermodal interference fringes are modulated in contrast when the sensor is powered with depolarized light. We determined the sensitivity coefficients for temperature and strain corresponding to fringe displacement and contrast variations. These coefficients are equal respectively for strain $-2.51 \mathrm{~nm} / \mathrm{mstrain}$, $-0.02561 / \mathrm{mstrain}$ and for temperature $16.7 \mathrm{pm} /{ }^{\circ} \mathrm{C}$ and $5.74 \times 10^{-5} 1 /{ }^{\circ} \mathrm{C}$. As the sensitivity matrix is well conditioned, the proposed sensor can be used for simultaneous measurements of temperature and strain.

The work presented in this paper was partially carried out with support of the Polish Ministry of Science and Education under grant NN 505560 439, a bilateral grant for cooperation between Poland and Portugal, and a statutory grant at Wroclaw University of Technology. G. StatkiewiczBarabach, J. Olszewski and W. Urbanczyk acknowledge support of the Foundation for Polish Science program MISTRZ.

\section{References}

1. H. Y. Fu, Chuang Wu, M. L. V. Tse, L. Zhang, K.-C. Davis Cheng, H. Y. Tam, B.-O. Guan, and C. Lu, "High pressure sensor based on photonic crystal fiber for downhole application," Appl. Opt. 49, 2639-2643 (2010).

2. X. Dong, H. Y. Tam, and P. Shum, "Temperature-insensitive strain sensor with polarization-maintaining photonic crystal fiber based Sagnac interferometer," Appl. Phys. Lett. 90, 151113 (2007).

3. O. Frazăo, J. M. Baptista, and J. L. Santos, "Temperatureindependent strain sensor based on a Hi-Bi photonic crystal fiber loop mirror," IEEE Sens. J. 7, 1453-1455 (2007).

4. T. M. Monro, W. Belardi, K. Furusawa, J. C. Baggett, N. G. R. Broderick, and D. J. Richardson, "Sensing with microstructured optical fibres," Meas. Sci. Technol. 12, 854-858 (2001).

5. J. M. Fini, "Microstructure fibres for optical sensing in gases and liquids," Meas. Sci. Technol. 15, 1120-1128 (2004). 
6. M. Hautakorpi, M. Mattinen, and H. Ludvigsen, "Surfaceplasmon-resonance sensor based on three-hole microstructured optical fiber," Opt. Express 16, 8427-8432 (2008).

7. T. Ritari, J. Tuominen, H. Ludvigsen, J. Petersen, T. Sørensen, T. Hansen, and H. Simonsen, "Gas sensing using air-guiding photonic bandgap fibers," Opt. Express 12, 4080-4087 (2004).

8. O. Frazão, J. L. Santos, F. M. Araújo, and L. A. Ferreira, "Optical sensing with photonic crystal fibers," Laser Photon. Rev. 2, 449-459 (2008).

9. G. Statkiewicz, T. Martynkien, and W. Urbanczyk, "Measurements of modal birefringence and polarimetric sensitivity of the birefringent holey fiber to hydrostatic pressure and strain," Opt. Commun. 241, 339-348 (2004).

10. J. Villatoro, V. P. Minkovich, V. Pruneri, and G. Badenes, "Simple all-microstructured-optical-fiber interferometer built via fusion splicing," Opt. Express 15, 1491-1496 (2007).

11. W. J. Bock, T. Eftimov, P. Mikulic, and J. Chen, "Novel fiber sensor based on in-line core-cladding intermodal interferometer and photonic crystal fiber," in Proceedings of XIX IMEKO World Congress Fundamental and Applied Metrology (IMEKO, 2009), pp. 65-68.

12. Y. Jung, S. Lee, B. H. Lee, and K. Oh, "Ultracompact in-line broadband Mach-Zehnder interferometer using a composite leaky hollow-optical-fiber waveguide," Opt. Lett. 33, 2934-2936 (2008).

13. J. Villatoro, V. Finazzi, G. Badenes, and V. Pruneri, "Highly sensitive sensors based on photonic crystal fiber modal interferometers," J. Sensors 2009, 747803 (2009).
14. V. Minkovich, J. Villatoro, D. Monzon-Hernandez, S. Calixto, A. Sotsky, and L. Sotskaya, "Holey fiber tapers with resonance transmission for high-resolution refractive index sensing," Opt. Express 13, 5087-5092 (2005).

15. H. Y. Choi, M. J. Kim, and B. H. Lee, "All-fiber Mach-Zehnder type interferometers formed in photonic crystal fiber," Opt. Express 15, 5711-5720 (2007).

16. G. Statkiewicz-Barabach, J. Olszewski, M. Napiorkowski, G. Golojuch, T. Martynkien, K. Tarnowski, W. Urbanczyk, J. Wojcik, P. Mergo, M. Makara, T. Nasilowski, F. Berghmans, and $\mathrm{H}$. Thienpont, "Polarizing photonic crystal fiber with low index inclusion in the core," J. Opt. 12, 075402 (2010).

17. M. Szpulak, W. Urbanczyk, E. Serebryannikov, A. Zheltikov, A. Hochman, Y. Leviatan, R. Kotynski, and K. Panajotov, "Comparison of different methods for rigorous modeling of photonic crystal fibers," Opt. Express 14, 5699-5714 (2006).

18. A. W. Snyder and J. D. Love, Optical Waveguide Theory (Chapman and Hall, 1983).

19. P. Hlubina, "White-light spectral interferometry to measure intermodal dispersion in two-mode elliptical-core optical fibres," Opt. Commun. 218, 283-289 (2003).

20. J. Villatoro, V. P. Minkovich, and D. Monzon-Hernandez, "Temperature-independent strain sensor made from tapered holey optical fiber," Opt. Lett. 31, 305-307 (2006).

21. J. Villatoro, V. Finazzi, V. P. Minkovich, V. Pruneri, and G. Badenes, "Temperature-insensitive photonic crystal fiber interferometer for absolute strain sensing," Appl. Phys. Lett. 91, 091109 (2007). 